\title{
Comprehensive assessment of Power Demand Side Management (PDSM) based on PCA-fuzzy synthetic evaluation
}

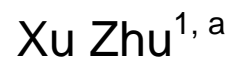 \\ ${ }^{1}$ School of Electrical and Electronic Engineering, North China Electric Power University, Baoding \\ 071003, China; \\ a974887035@qq.com
}

Keywords: power demand side management, principal components analysis (PCA), fuzzy synthetic evaluation

\begin{abstract}
Integrated with the characteristics of comprehensive assessment, comprehensive evaluation index system of power demand side management (PDSM) is established. A quantitative evaluation model of PDSM implementation is proposed. In the model, index weight is calculated through principal components analysis (PCA) to grade each parameter based on fuzzy synthetic evaluation. Analysis results of PDSM in Beijing illustrate the feasibility and validity of the presented method.
\end{abstract}

\section{Introduction}

PDSM is a kind of power consumption management activity, which optimizes power consumption way, economizes energy and reduces cost. It focuses on load management, integrated resource planning and energy efficiency. The detailed content includes peak shaving, valley filling, load transfer, power saving and variable load [1-3]. The means of PDSM are divided into four parts: technical means, economic means, guide means and administrative means[4].

The previous evaluation index of PDSM implementation mainly aims at energy conservation and pollution reduction or market benefit, which is incomprehensive and unsystematical[5-6]. Traditional PDSM comprehensive assessment system is based on AHP (analytic hierarchy process), but the qualitative measure is influenced by human factors greatly[7-8]. A more objective method must be adopted.

This paper presents a model of PDSM comprehensive assessment combining subjective and objective index. Quantitative index can be obtained from data and research; qualitative index needs evaluating though fuzzy statistical method. All index weight is given by PCA and the distribution vector is ensured. Using multi-level fuzzy comprehensive evaluation, the assessment of PDSM is attained. PDSM evaluation of Beijing is carried out, and the results show that the model is practicable.

\section{Comprehensive evaluation index system of PDSM}

There are 23 evaluation index, $X_{1}, X_{2}, \cdots, X_{23}$ of PDSM, which forms the table.1[7-8].

Typical ones are selected for introduction and calculation is below.

A. Quantitative index

$X_{1}$ : Avoidable capacity is the saving generation capacity during peak load period.

$$
F_{N}=\Delta N_{\gamma} \cdot I_{j} \quad \Delta N_{\gamma}=e_{\gamma} \Delta P_{f}
$$

Above, $F_{N}$ is the cost of avoidable peak load capacity, $I_{j}$ is the unit investment of generating set, $\Delta N_{\gamma}$ is the avoidable peak load capacity, $\Delta P_{f}$ is the avoidable power, $e_{\gamma}$ is the coefficient of avoidable capacity, which are 1.32-1.63 generally.

$X_{17}: S_{2}$ emissions are the reduced $\mathrm{SO}_{2}$ emissions of power plants.

$$
A_{\mathrm{SO}_{2}}=\lambda_{\mathrm{SO}_{2}} \cdot B \quad \lambda_{\mathrm{SO}_{2}}=\alpha_{\mathrm{s}} \cdot \beta_{\mathrm{SO}_{2}} \cdot \gamma_{\mathrm{s}}
$$


Above, $A_{\mathrm{SO}_{2}}$ are the reduced emissions of $\mathrm{SO}_{2}, \lambda_{\mathrm{SO}_{2}}$ is the coefficient of reduced emissions, $\alpha_{s}$ is the sulfur content rate of coal, $\beta_{\mathrm{SO}_{2}}$ is the conversion factor from $S$ to $\mathrm{SO}_{2}, \gamma_{S}$ is release rate of $S$.

For Chinese coal-fired power plants, $\alpha_{\mathrm{s}}=1.0 \%-1.2 \%, \quad \beta_{\mathrm{SO}_{2}}=2, \gamma_{\mathrm{s}}=0.85$.

B. Qualitative index

It's hard to obtain the relationship between client satisfaction $X_{10}$, Sustainable development $X_{21}$, Economic development $X_{22}$ or Government image $X_{23}$ and PDSM by formula. Adopting 5-point scale, experts mark implementation of PDSM.

Table.1 Comprehensive evaluation index system of PDSM

\begin{tabular}{llcl}
\hline \multicolumn{1}{c}{ Generation side } & \multicolumn{1}{c}{ Grid side } & Big user & \multicolumn{1}{c}{ Society } \\
\hline & \multicolumn{1}{c}{ Input } & & Environmental benifits \\
& $X_{5}$ Reduced power sale & & $X_{16} \mathrm{CO}_{2}$ emissions \\
& $X_{6}$ Cost of advocacy & Input & $X_{17} \mathrm{SO}_{2}$ emissions \\
$X_{1}$ Avoidable capacity & Benifits & $X_{12}$ Equipment & $X_{18} \mathrm{NO}_{x}$ emissions \\
$X_{2}$ Avoidable fuel & $X_{7}$ Grid investment & $X_{13}$ Cost of operation & $X_{19}$ Solid waste emissions \\
$X_{3}$ Unit start and stop & $X_{8}$ Maintenance & Benifits & $X_{20}$ Wastewater emissions \\
$X_{4}$ Pollution compensation & $X_{9}$ Reliability & $X_{14}$ Payback period & Development benefits \\
& $X_{10}$ Client satisfaction & $X_{15}$ Cost of brownout & $X_{21}$ Sustainable development \\
& $X_{11}$ Load rate & & $X_{22}$ Economic development \\
& & & $X_{23}$ Government image \\
\hline
\end{tabular}

\section{Comprehensive evaluation model of PDSM}

A. Determination of index weight

PCA is used to determinate the weight of PDSM index[9]. Calculating the linear combination of the $p$ index, new comprehensive index can be attained. The first principal component $F_{1}$ is of the maximum variance, and then is $F_{2}$. In this way, $p$ principal components can be constructed. Cumulative contribution rate reaches $85 \%$, which indicates principal components contain main information of previous index. PCA mathematical model is as follows:

$$
\begin{gathered}
F_{1}=a_{11} x_{1}+a_{21} x_{2}+\cdots+a_{p 1} x_{p} \\
F_{2}=a_{12} x_{1}+a_{22} x_{2}+\cdots+a_{p 2} x_{p} \\
\vdots \\
F_{p}=a_{1 m} x_{1}+a_{2 m} x_{2}+\cdots+a_{p m} x_{p}
\end{gathered}
$$

Where $a_{1 i}, a_{2 i}, \cdots, a_{p i}(i=1, \cdots, m)$ is the eigenvectors corresponding to eigenvalues, $x_{1}, x_{2}, \cdots, x_{p}$ is the variables of standardized data-processing.

By weighted average method, the coefficient of index in each principal components can be changed into that of comprehensive score model. The index weight is the coefficient of comprehensive score model.

B. Multi-level fuzzy comprehensive evaluation

As for the index of large number, multi-level fuzzy comprehensive evaluation is adopted. The explaination to second-level fuzzy comprehensive evaluation is given as follows[7-8]:

The factor set $\mathbf{U}$ of $m$ dimension is divided into several subsets $\mathbf{U}_{i}=\left\{\mathbf{U}_{i 1}, \mathbf{U}_{i 2}, \cdots, \mathbf{U}_{i n}\right\},(i=1,2, \cdots, k)$. The comments and corresponding membership of evaluation set $\mathbf{V}=\left\{v_{1}, v_{2}, \cdots, v_{n}\right\}$ is below: 


$$
P=\left\{\begin{array}{c}
0.85 \leq \mu \leq 1.0(\text { excellent }), 0.70 \leq \mu \leq 0.85(\text { good }), 0.60 \leq \mu \leq 0.70 \text { (ordinary }) \\
0.40 \leq \mu \leq 0.60(\text { poor }), 0 \leq \mu \leq 0.40(\text { bad })
\end{array}\right\}
$$

The comprehensive evaluation of $\mathbf{U}_{i}: \mathbf{B}_{i}=\mathbf{A}_{i} o \mathbf{R}_{i}=\left(b_{i 1}, b_{i 2}, \cdots, b_{i m}\right)$

Where, $\mathbf{A}_{i}=\left\{a_{i 1}, a_{i 2}, \cdots, a_{i n}\right\}$ is the weight vector set calculated by PCA, $\mathbf{R}_{i}$ is the single factor evaluation matrix of $\mathbf{U}_{i}$.

The single factor evaluation matrix of $\mathbf{U}=\left\{u_{1}, u_{2}, \cdots, u_{k}\right\}: \mathbf{R}=\left[\begin{array}{c}\mathbf{B}_{1} \\ \mathbf{B}_{2} \\ \vdots \\ \mathbf{B}_{k}\end{array}\right]=\left[\begin{array}{cccc}b_{11} & b_{12} & \cdots & b_{1 m} \\ b_{21} & b_{22} & \cdots & b_{2 m} \\ \vdots & \vdots & \vdots & \vdots \\ b_{k 1} & b_{k 2} & \cdots & b_{k m}\end{array}\right]$

\section{Example test of PDSM comprehensive evaluation system}

According to the research index data of Beijing in 2000-2015, PDSM implementation is analysed and evaluated.

The extraction of principal components is shown in table.2.

Table.2 The extraction information of principal components

\begin{tabular}{ccc}
\hline Component & Contribution rate(\%) & Cumulative contribution rate(\%) \\
\hline 1 & 24.914 & 24.914 \\
2 & 17.913 & 42.827 \\
11 & 21.554 & 64.381 \\
21 & 22.682 & 87.063 \\
\hline
\end{tabular}

The cumulative contribution rate of index $X_{1}, X_{2}, X_{11}$ and $X_{21}$ is $87.063 \%$, which shows principal components contain Most information of PDSM index.

The calculated results of 4 principal components is below:

$$
\begin{aligned}
& F_{1}=0.867 x_{1}+0.638 x_{2}+0.549 x_{3}-0.297 x_{4} \cdots+0.543 x_{20}+0.414 x_{21}+0.318 x_{22}+0.413 x_{23} \\
& F_{2}=0.281 x_{1}+0.314 x_{2}-0.372 x_{3}+0.546 x_{4} \cdots+0.462 x_{20}+0.371 x_{21}-0.122 x_{22}+0.154 x_{23} \\
& F_{3}=0.422 x_{1}+0.217 x_{2}+0.661 x_{3}+0.542 x_{4} \cdots-0.232 x_{20}+0.274 x_{21}+0.105 x_{22}-0.021 x_{23} \\
& F_{4}=0.181 x_{1}+0.632 x_{2}+0.142 x_{3}+0.892 x_{4} \cdots+0.724 x_{20}+0.831 x_{21}+0.278 x_{22}+0.108 x_{23}
\end{aligned}
$$

comprehensive score model is as follows:

$Y=0.463 x_{1}+0.448 x_{2}+0.253 x_{3}+0.375 x_{4} \cdots+0.367 x_{20}+0.454 x_{21}+0.142 x_{22}+0.175 x_{23}$

The coefficient of comprehensive score mode is index weight of PDSM. To present simply, the percentage of index weight is shown in table.3.

Table.3 PDSM index weight

\begin{tabular}{cccccccccccc}
\hline$X_{1}$ & $X_{2}$ & $X_{3}$ & $X_{4}$ & $X_{5}$ & $X_{6}$ & $X_{7}$ & $X_{8}$ & $X_{9}$ & $X_{10}$ & $X_{11}$ & $X_{12}$ \\
\hline 0.082 & 0.079 & 0.045 & 0.066 & 0.041 & 0.043 & 0.030 & 0.043 & 0.021 & 0.014 & 0.066 & 0.050 \\
\hline$X_{13}$ & $X_{14}$ & $X_{15}$ & $X_{16}$ & $X_{17}$ & $X_{18}$ & $X_{19}$ & $X_{20}$ & $X_{21}$ & $X_{22}$ & $X_{23}$ & \\
\hline 0.037 & 0.034 & 0.022 & 0.015 & 0.069 & 0.057 & 0.032 & 0.065 & 0.080 & 0.025 & 0.031 & \\
\hline
\end{tabular}

The results of fuzzy comprehensive evaluation is in the following table.4. 
Table.4 The results of PDSM comprehensive evaluation

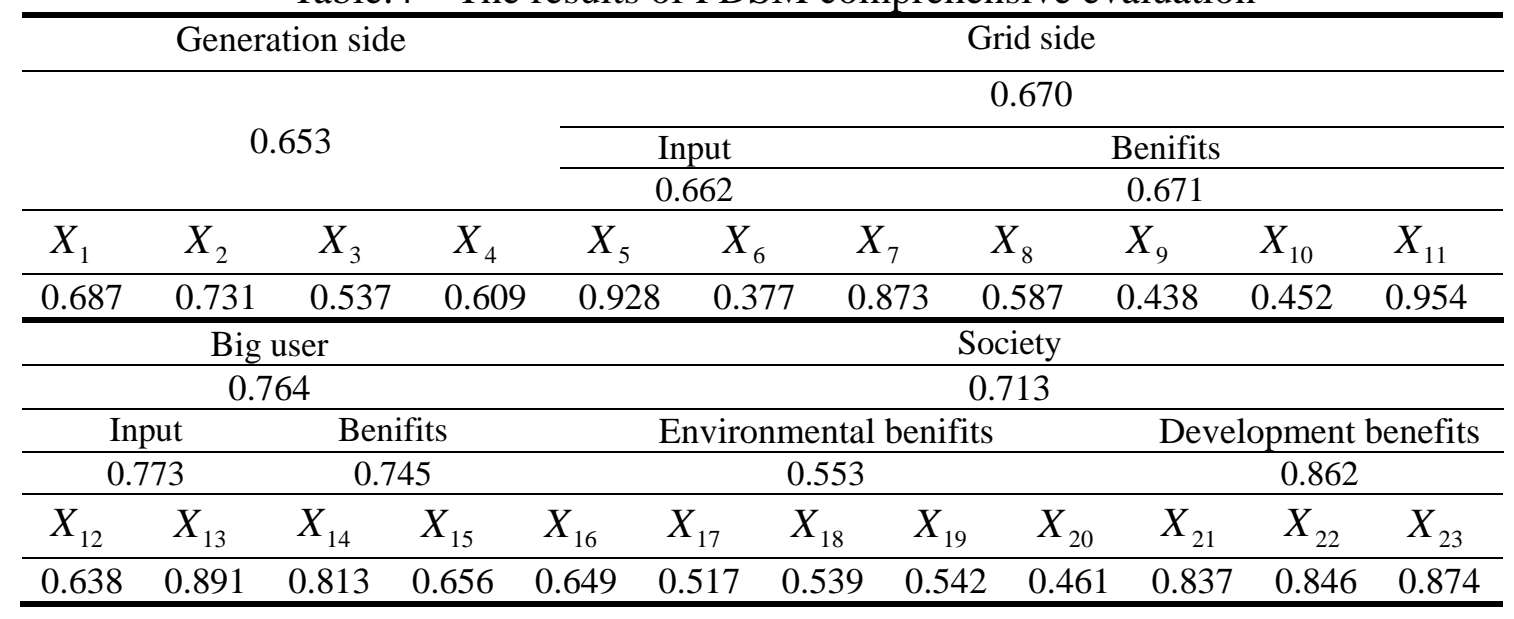

Evaluating the first layer of PDSM evaluation index system, final membership degree in Beijing is 0.703 , whose comment is good. In order to obtain clearer evaluation, the result illustrated with a quadrant chart is shown in chart.1[9].

The index weight represents the importance, and the membership represents the evaluation results. On the basis of the average weight and average membership, the chart can be divided into four quadrant.

The index in first quadrant is of high importance and high membership (High attention). Index $X_{1}, X_{2}, X_{11}$ and $X_{21}$ is in this quadrant, which deserves to be supported; The index in second quadrant is of high importance but low membership (Priority improvement). Index $X_{3}, X_{4}, X_{12}, X_{17}, X_{18}$ and $X_{20}$ is in this quadrant. It is necessary to take the improvement measurements; The index in third quadrant is of low importance and low membership(Unimportant area). Index $X_{6}, X_{8}, X_{9}, X_{10}, X_{15}, X_{16}$ and $X_{19}$ is in this area. These index only needs paying attention to the change of importance; The index in fourth quadrant is of low importance and high membership (Maintain advantage). Index $X_{5}, X_{7}, X_{13}, X_{14}, X_{22}$ and $X_{23}$ is in this quadrant, the resources of these index should be transferred to other more important index, such as the index in second quadrant. Obviously, the strengths and weaknesses of PDSM in Beijing is found out to refocus the work.

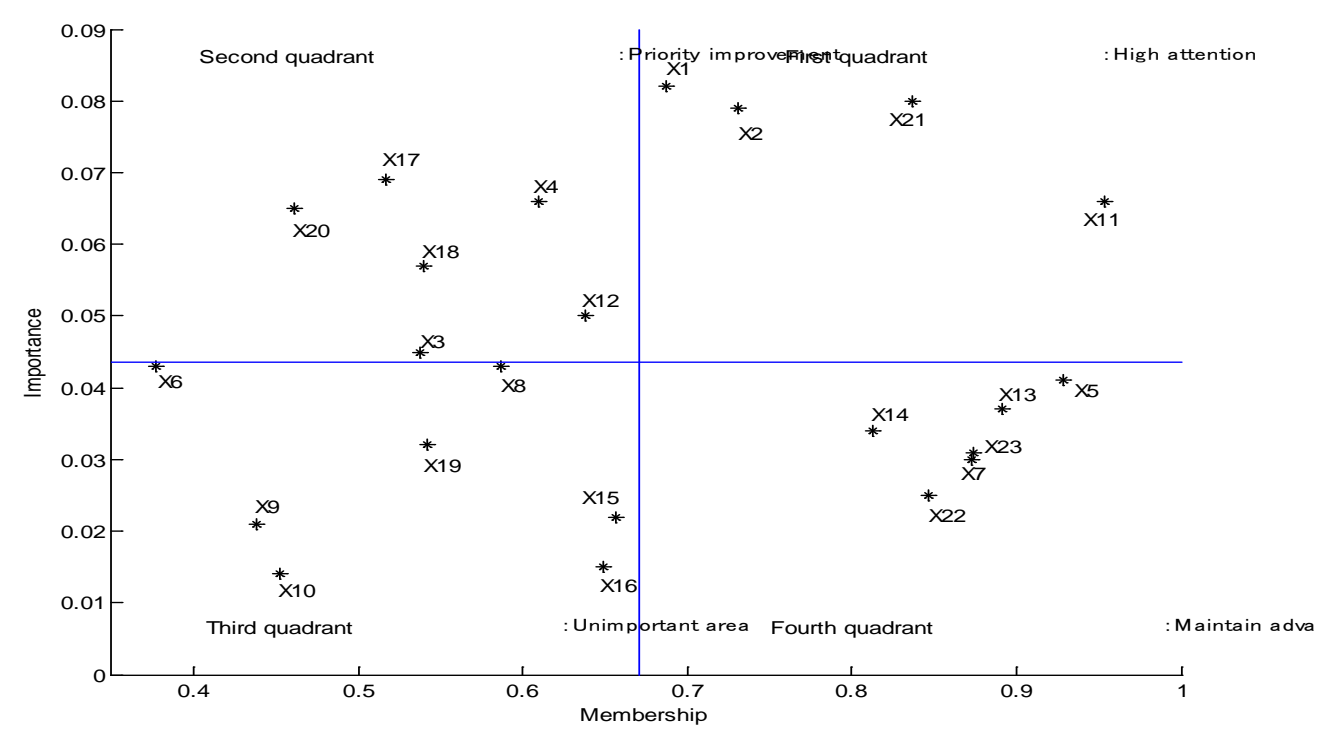

Chart.1 The evaluation of PDSM index 


\section{Summary}

This paper establishes the comprehensive evaluation index system of PDSM. Combining PCA with multi-level fuzzy evaluation, comprehensive evaluation model of PDSM is built. All index weight and membership is obtained. The classification of index makes the assessment easier. The results of PDSM evaluation has important reference value for current implementation and future management work.

\section{References}

[1] H.G. Lu: Control Technique for Power Load \& Application in DSM, vol. 18 (2011) no. 1, p. 128.

[2] N. Su: Application Research for Demand Side Management of Beijing Chaoyang District (Master, North China Electric Power University, China 2008).

[3] P. Hu: The Study of Applying Power Demand Side Management for Southern Hebei Power Network (Master, North China Electric Power University, China 2006).

[4] L. Zhang: Research on Demand Side Management of Binzhou City (Master, Shandong University, China 2010).

[5] J.H. Chen, J.Y. Xu: Means to achieve the DSM Goals and Impact Factors Analysis of Grid Corporations, vol. 13 (2011) no. 2, p. 4-6.

[6] P.F. Wang: Improvement on DSM Project Evaluation Model under Market Environment, vol. 10 (2008) no. 1, p. 19-21.

[7] Z.P. Qi: Research on Assessment for Power Demand Side Management (Master, North China Electric Power University, China 2004).

[8] X.L. Tian, H.X. Cheng, C. Liu: The Application of Fuzzy Evaluation in PDSM Assessment, (2008) no. 2, p. 192-194.

[9] W.L. Zhang: The Application of the Key Elements Analysis in Ascertaining the Satisfaction Weight, vol. 6 (2006) no. 1, p. 18-22. 